\title{
The use of pre-cannulation local anaesthetic and factors affecting pain perception in the emergency department setting
}

\author{
T Harris, P A Cameron, A Ugoni
}

\begin{abstract}
Study objective-To determine whether the use of subcutaneous local anaesthetic (lignocaine) is associated with a reduction in cannulation pain in the emergency department setting.

Methods-Patients over 18 with a Glasgow Coma Score (GCS) of 15 and conversational English were allocated into one of three groups: Group 1 were cannulated after routine skin preparation; Group 2 received $1 \%$ lignocaine $0.1 \mathrm{ml}$ via a 27 gauge needle and diabetic syringe before cannulation; Group 3 were injected as for Group 2 but saline was substituted for lignocaine. The cannulator and subject were blinded to the ampoule. The pain was measured using a $100 \mathrm{~mm}$ visual analogue scale.

Setting-A large urban university hospital emergency department.

Results-366 patients were recruited and the data on 322 analysed. Those receiving lignocaine before cannulation reported lower pain scores $(1.9 \mathrm{~cm})$ than the saline $(4.1 \mathrm{~cm})$ or immediate cannulation (3.6 cm) groups, $p<0.0001$. Other factors such as the experience of cannulator, patient characteristics, the presence of a painful underlying condition and cannula size did not effect pain scores.

Conclusion-The use of lignocaine before cannulation reduced cannulation pain in the emergency department setting. Other factors examined did not influence pain perception.

(Emerg Med f 2001;18:175-177)
\end{abstract}

Keywords: anaesthesia; lignocaine; lidocaine

Emergency

Department, Royal

Melbourne Hospital, PO Box 2009,

Parkville, Victoria

3050, Australia

T Harris

P A Cameron

The University of Melbourne, Parkville, Melbourne

A Ugoni

Correspondence to: Professor Cameron (peter.cameron@mh.org.au)

Accepted for publication 26 April 2000
Local anaesthetic to relieve the pain of intravenous cannulation is widely used in paediatric and some anaesthetic practice..$^{1-7}$ Various methods are used including topical ethyl chloride, eutetic mixture of local anaesthetic (EMLA) and intradermal or subcutaneous (SC) lignocaine (lidocaine) ${ }^{8}$ All have been shown to significantly reduce canulation pain. ${ }^{458}$ SC lignocaine injection has been shown to be less painful than insertion of 18 , 20 and 22 gauge cannulas. ${ }^{6}$ Post-cannulation insertion site pain may be abolished by the use of local anaesthetic (LA). ${ }^{5}$ Despite the evidence favouring the use of LA, many clinicians fail to do so, ${ }^{7}$ believing the pain of cannulation to be minimal or not worth the time and expense of LA.
A further reason for emergency clinicians not using LA is that most studies have been performed on non-emergency patients. The majority of studies have been performed on children and preoperative adults cannulated in the operating room awaiting routine surgery, who are likely to be anxious about the surgery and related procedures. It is postulated that the anxiety of emergency department (ED) patients is different, in that it is often related to the presenting complaint compared with the imminent procedure. More patients in the ED will be in pain on attendance. These factors may change the perception of pain.

Although lignocaine is itself painful to inject, discomfort may be minimised by warming to $42^{\circ} \mathrm{C}^{9}$ and by alkalisation. ${ }^{10}{ }^{11}$ However, buffered lignocaine has a shelf life of less than one week. ${ }^{12}$ Lignocaine and mepivicaine have been shown to be less painful than other LAs for SC injection. ${ }^{13}{ }^{14}$

EMLA cream has been used extensively in paediatrics, but needs $30-60$ minutes to create effective analgesia ${ }^{811}$ and thus is not suitable for many emergency conditions. SC lignocaine $1 \%$ is effective quickly and is widely available in nearly all EDs.

This study was designed to compare the pain of cannulation with or without LA ( $1 \%$ SC lignocaine) in the ED and to examine factors affecting the perception of pain by the subject (patient)

\section{Methods}

SETTING

The Royal Melbourne Hospital Emergency Department is a tertiary level university teaching ED, with annual census of 42000 patients.

The following patients were included in the study:

- Age over 18

- ED presentation requiring cannulation

- Non-critical condition

- GCS 15

- Conversational English

Exclusion criteria:

- Allergy to lignocaine

- Dementia, acute brain syndrome

- Drugs ingested that may change pain perception (including opioids and alcohol)

- Refusal to participate

- Potentially difficult cannulation

Patients were also excluded from statistical analysis if there were incomplete data or if more than two attempts were made at cannulation.

Patients were randomised according to sequential number allocation. 
Three groups were compared:

(1) IV cannulation with no anaesthetic.

(2) IV cannulation with $1 \%$ lignocaine $0.1 \mathrm{ml}$ SC 30 seconds before cannulation using 27 gauge needle and insulin syringe.

(3) IV cannulation with normal saline $0.9 \%$ $0.1 \mathrm{ml} \mathrm{SC}$ using the same equipment as (2) (above).

Nursing and medical staff (interns, residents, registrars and specialists) working within the ED performed intravenous cannulation according to the following protocol.

The staff member performing the cannulation chose an envelope containing a plain language statement explaining the trial to the patient, a consent form and a visual analogue pain scale (see below). One third of envelopes contained a 27 gauge insulin syringe and a masked vial of lignocaine, one third contained the syringe and a masked vial of saline and the remainder contained neither syringe nor vial.

An explanation was given to the patient, consent obtained and the skin prepared with chlorhexidine. In group 1 , a cannula chosen by the staff member was inserted, in groups 2 and $30.1 \mathrm{ml}$ of either lignocaine $1 \%$ or saline was injected by slow SC injection. The cannula was inserted after 30 seconds.

In all groups the patient was then asked to mark the pain on a $100 \mathrm{~mm}$ visual analogue scale (VAS) as recommended by Ho et al. ${ }^{15}$

DATA COLLECTED

- Pain value on visual pain assessment scale (above).

- Age, sex and diagnosis of subject.

- Job classification of cannulator (nurse, intern, resident, registrar, specialist).

- The presence or absence of pain in the subject at the time of cannulation.

Because of the nature and non-normal shape of the data non-parametric analysis was used (Mann-Whitney when comparing two groups and Kruskal-Wallis more than two groups).

\section{Results}

Altogether 366 subjects were recruited and data were analysed on 322, 105 of who received lignocaine, 105 received saline and 112 were cannulated with no injection. Of the 44 patients excluded, 17 were excluded because two or more cannulation attempts were made and the others were excluded because of incomplete data. Mean pain scores are presented in tables 1-3.

CANNULATION PAIN AND LA (TABLE 1)

Multiple comparison after use of the KruskalWallis test demonstrated that the lignocaine group had a significantly lower pain score than the other two groups $(\mathrm{p}<0.0001)$. There was

Table 1 Pain score compared with anaesthetic use

\begin{tabular}{lccc}
\hline & $\begin{array}{l}\text { Local } \\
\text { anaesthetic }\end{array}$ & Saline & Control \\
\hline Number & 105 & 105 & 112 \\
Mean pain scale (cm) & 1.9 & 4.1 & 3.6 \\
Median pain score (cm) & 1.5 & 4.0 & 3.5 \\
SD & 1.30 & 1.97 & 1.98 \\
\hline
\end{tabular}

Table 2 Pain score by cannula size

\begin{tabular}{lcl}
\hline Cannula size & Number in sample & Pain score (mean) cm \\
\hline 16 & 11 & 3.6 \\
18 & 104 & 3.0 \\
20 & 190 & 3.4 \\
22 & 14 & 2.5 \\
\hline
\end{tabular}

Table 3 Pain score by operator

\begin{tabular}{lcl}
\hline Investigator grade & Number & Pain score (mean) cm \\
\hline Consultant & 14 & 3.3 \\
Nurse & 25 & 2.9 \\
Intern & 48 & 3.8 \\
Registrar & 193 & 3.0 \\
SRMO & 31 & 3.0 \\
JRMO & 7 & 4.0 \\
\hline
\end{tabular}

no significant difference between the two control groups. The mean pain values were slightly lower than most other studies. ${ }^{8}$

CANNULATION PAIN BY CANNULA SIZE (TABLE 2) There was no significant difference in pain score by cannula size, however there were only 11 patients with 16 gauge and 14 patients with 22 gauge cannulas $(\mathrm{p}=0.377$, Kruskal-Wallis test).

\section{PAIN SCORE BY CANNULATOR EXPERIENCE} (TABLE 3)

There was no significant difference between different groups of cannulators by experience, (Kruskal-Wallis test, $\mathrm{p}=0.3083$ ).

CANNULATION PAIN AND THE PRESENCE OF PAIN FROM THE UNDERLYING MEDICAL CONDITION Pain from an underlying medical condition at the time of cannulation was absent for 151 patients and present in 170 patients. There was no significant difference between the two groups ( $p=0.6637$, Mann-Whitney test). For comparison the means were 3.1 and 3.3 respectively.

\section{CANNULATION PAIN AND PATIENT} CHARACTERISTICS

Cannulation pain and the sex of the patient were analysed. There was no significant difference between the male and female groups ( $p=0.5776$, Mann-Whitney test). For comparison the mean pain score for man was 3.2 and for women was 3.1. Cannulation pain and age of patient were compared using the Spearman rank correlation. There was no significant difference $(0.0410, \mathrm{p}=0.4850)$.

NUMBER OF CANNULATION ATTEMPTS (TABLE 4) The failed cannulation rate was examined and found to be 17 of 111 in the LA group, 19 of 112 in the saline group, and 12 of 116 in the direct insertion group, which were not significantly different from each other (Fisher's exact

Table 4 Number of cannulation attempts

\begin{tabular}{lcl}
\hline & One attempt & Two or more attempts \\
\hline Local anaesthetic & 94 & 17 \\
Normal saline & 93 & 19 \\
Nil & 104 & 12 \\
\hline
\end{tabular}


test $p=0.314)$. Only five of these were in the registrar or consultant group.

\section{Discussion}

The objective of this study was to examine the effect of SC LA (1\% lignocaine 30 seconds before cannulation) on the pain of intravenous cannulation. Two control groups were used, firstly using normal saline in place of lignocaine and secondly immediate cannulation. The distribution of pain scores for the controls were not sigificantly different, while the lignocaine group had a significantly different and lower pain score. This is likely to be clinically significant, as the LA group had a mean pain score of $1.9 \mathrm{~mm}$, which was $2.2 \mathrm{~mm}$ and $1.7 \mathrm{~mm}$ lower than controls (saline group mean $4.1 \mathrm{~mm}$ and direct insertion group 3.6 $\mathrm{mm})$. Todd et al found a $13 \mathrm{~mm}$ difference on a $100 \mathrm{~mm}$ VAS to be the minimum clinically significant value. ${ }^{16}$ This study demonstrates that lignocaine lowers cannulation pain for adults in an ED setting.

The study also examined other factors affecting cannulation pain.

Cannula size: inserting a larger cannula would intuitively be expected to produce more pain than a smaller one, and previous studies have supported this. ${ }^{45}$ However, in this study there was no difference in the pain associated with $16,18,20$ or 22 gauge cannula. It is probable that the explanation for this is the small number of 16 gauge and 22 gauge cannulas used in this study.

Cannulator experience: similarly, it was expected that more experienced operators would produce less cannulation pain, this was not shown to be so. This may be explained by two factors; firstly, all medical staff had performed many cannulations before the study (medical and nursing students were excluded and more junior nurses do not practise cannulation in this hospital), thus the learning curve was probably complete for most/all participants. Secondly, any subject requiring more than two attempts at cannulation was withdrawn from the study.

Associated painful medical condition: it was also postulated that patients in pain would score a higher pain score reflecting their higher arousal and anxiety. This was not supported by the data. It thus seems that the presence or absence of pain did not change the patient's cannulation pain perception.

Patient characteristics: finally, the effect of age and sex on pain scores was examined. Anecdotally medical staff felt that older men seemed less distressed by cannulation and younger women more so. Previous studies have found a complex relation between sex and pain, some suggesting a difference with men reporting less pain, others have contradicted this and some have found no differences. ${ }^{41718}$ Our study found the mean pain scores for men and for women were not significantly different, and there was no correlation with age. One explanation for this might be that the way we express pain is culturally dependent and varies with age and sex even though the level of pain perceived is constant.

The use of LA also seems not to affect the success rate of cannulation, there being no significant difference between the three groups. This issue is often raised as a reason for not using LA however most staff found that cannulation difficulty was not increased once they had practised cannulation with LA. There were a surprisingly small number of patients in the group with three or more attempts at cannulation. The small size of this group most probably reflects the exclusion of patients in whom a difficult cannulation was anticipated and the relative experience of the cannulators.

This study has found that the slow subcutaneous injection of $0.1 \mathrm{ml}$ of lignocaine $1 \%, 30$ seconds before cannulation significantly reduces the associated pain levels. Furthermore, we found that perception of pain is not dependent on the seniority of the operator, or the characteristics of the patient. There is now convincing evidence that IV cannulation in the ED should be preceded by local anaesthetic unless there is some overriding clinical urgency.

1 Keep PJ, Jenkins JR. From the other end of the needle. The patient's experience of routine anaesthesia. Anaesthesia 990;45:679-82.

2 Hopkins CS, Buckley CJ, Bush GH. Painfree injections in infants; use of lidocaine-prilocaine cream to prevent pain at intravenous induction of anaesthesia in 1-8 year old children. Anesthesiology 1982;57:326-8.

3 Soliman IE, Broadman LM, Hannallah RJ, et al. Comparison of analgesia effects of EMLA to intradermal lidocaine infiltration prior to venous cannulation in unpremedicated children. Anesthesiology 1988;68:804-6.

4 Van den Berg AA, Rama Pabhu NV. Rationalizing venepuncture pain: Comparison of ligocaine injection, Butterfly (21 and 23 gauge) and venflon (20 gauge). Anaesth Intensive Care 1995;23:165-7.

5 Langman BT, Harrison DA. Local anaesthetic; does it really reduce the pain of insertion of all sizes of venous cannula? Anaesthesia 1992;47:890-1.

6 Harrison N, Langham BT., Bogod DG. Appropriate use of local anaesthetic for venous cannulation. Anaesthesia 1992; 47:210-12.

7 Criswell J, Gauntlett IS. Pain on intradermal injection with lignocaine - the effect of concentration. Anaesthesia 1991; 46:691-2.

8 Selby IR, Bowles BJM. Analgesia for venous cannulation: a comparison of E.M.L.A, lignocaine, ethyl chloride and nothing. I R Soc Med 1995;88:264-7.

9 Waldbillig DK, Quin JV, Stiell IG, et al. Randomised double-blind controlled trial comparing room temperature and heated lidocaine for digital nerve block. Ann Emerg Med 1995;26:677-81.

10 Nuttal GA, Barrett MR, Smith RL, et al. Establishing intravenous access: a study of local anaeshetic efficiency. Anesth Analg 1993;77:950-3.

11 Steinbrook RA, Hughes N, Fanciullo G, et al Effects of alkalinization of lidocaine on the pain of skin infiltration and intravenous catheterization. F Clin Anesth 1993;5:456-8.

12 Bartfield ZM, Homer PJ, Fort DT. Buffered lidocaine as a local anaesthetic: an investigation of shelf life. Ann Emerg Med 1992;21:16-19.

13 Morris R, Mckay W, Mushlin P. Comparason of pain associated with intradermal and subcutaneous infiltration with various local anaesthetic solutions. Anesth Analg 1987;66: 1180-2.

14 Prien TH. Intradermal anaesthesia: comparison of several compounds. Infiltration pain of local an0aesthetic has been shown to be less for mepivicaine than lignocaine and more than both for procaine. Acta Anaesthesiol Scand 1994;38: 805-7.

15 Ho K, Spence J, Murphy M. Review of pain measurement tools. Ann Emerg Med 1996;27:427-31.

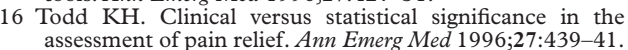

17 Van den Berg AA, Abeysekera RMMS. Rationalizing venous cannulation; patient factors and lignocaine efficiency. Anaesthesia 1993;48:84.

18 Nevin K. Influence of sex on pain assessment and management. Ann Emerg Med 1996;27:424-5. 